\title{
Evaluation of Bio-pesticides and Insecticides Against Brinjal Shoot and Fruit Borer (Leucinodes orbonalis Guenee) in Meghalawya of North-Eastern India
}

\author{
Sandip Patra*, N. S. Azad Thakur and D. M. Firake
}

Division of Crop Protection, ICAR Research Complex for North-Eastern Hill Region, Umiam, Meghalaya (793 103), India

Article History

Manuscript No. AR1650

Received in $6^{\text {th }}$ August, 2016

Received in revised form $29^{\text {th }}$ September, 2016

Accepted in final form $6^{\text {th }}$ October, 2016

\section{Correspondence to}

"E-mail: sandippatra47@gmail.com

\section{Keywords}

Bio-pesticides, Bacillus thuringiensis, azadirachtin, chlorantraniliprole, flubendiamide, Leucinodes orbonalis

\begin{abstract}
The field experiments were conducted at ICAR Research Complex for North Eastern Hill Region, Umiam, Meghalaya, India during 2011-2012 to evaluate some biopesticides and newly introduced insecticides against shoot and fruit borer of brinjal. The experiments were laid out in Randomized Block Design (RBD) with three replications. The treatments viz., azadirachtin $1 \mathrm{EC}\left(2 \mathrm{ml}^{-1}\right)$, karanjin $2 \mathrm{EC}\left(2 \mathrm{ml} \mathrm{l}^{-1}\right)$, anonin $1 \mathrm{EC}$ $\left(2 \mathrm{ml} \mathrm{l}^{-1}\right)$, Bacillus thuringiensis (Bt.) $\left(2 \mathrm{~g} \mathrm{l}^{-1}\right)$, emamectin benzoate $5 \mathrm{SG}\left(0.4 \mathrm{ml} \mathrm{l}^{-1}\right)$, flubendiamide $480 \mathrm{SC}\left(0.3 \mathrm{ml} \mathrm{l}^{-1}\right)$, chlorantraniliprole $18.5 \mathrm{SC}\left(0.4 \mathrm{ml} \mathrm{l}^{-1}\right)$, chlorpyriphos $20 \mathrm{EC}\left(2 \mathrm{ml} \mathrm{l}^{-1}\right)$ were applied thrice at fifteen days interval starting from initiation of shoot and fruit borer infestation. Results showed that chlorantraniliprole $(2.46 \%)$ was the best treatment in reducing the shoot infestation ( $81.88 \%$ reduction) which was statistically at par with flubendiamide (3.08\%) and emamectin benzoate $(3.76 \%)$ with $77.37 \%$ and $71.95 \%$ reduction over untreated control, respectively. The overall mean fruit infestation were also minimum in chlorantraniliprole $(5.76 \%)$ and flubendiamide $(5.93 \%)$ treated plots with $79.45 \%$ and $78.84 \%$ reduction over untreated control plots $(28.03 \%)$, respectively. Pooled data of two years experimental results indicated that highest marketable yield was recorded in chlorantraniliprole treated plots $(155.01 \mathrm{q}$ $\mathrm{ha}^{-1}$ ) followed by flubendiamide (149.50 q ha-1), emamectin benzoate (134.24 q ha-1) and chlorpyriphos (125.43 q ha-1). Among bio-pesticides, highest mean marketable yield was recorded in Bacillus thuringiensis treated plots (114.45 $\mathrm{q} \mathrm{ha}^{-1}$ ) followed by azadirachtin $\left(101.11 \mathrm{q} \mathrm{ha}^{-1}\right)$.
\end{abstract}

\section{Introduction}

Meghalaya is one of the biodiversity rich state of India in terms of flora and fauna. Varied altitude, topography, status of soil and climatic conditions favours high species richness and support different types of vegetation. The region is highly dynamic in case of weather, thus very suitable for multiplication of insect pests and their natural enemies. Brinjal or eggplant is one of the most important solanaceous vegetable grown all over the country. The area under brinjal cultivation in Meghalaya state is 0.96 thousand ha and production is 13.05 thousand $t$ with a productivity of only $13.59 \mathrm{mt} \mathrm{ha}^{-1}$ (Anonymous, 2014) which is much lower than national average of $19.1 \mathrm{mt} \mathrm{ha}^{-1}(\mathrm{NHB}, 2015)$. Insect pests and diseases are major limiting factor in brinjal productivity in this region. Several insect pests attack brinjal crop right from its nursery stage to harvesting (Regupathy et al., 1997); of which aphid (Aphis gossypii Glover), whitefly (Bemisia tabaci Lind.), jassid (Amrasca biguttula biguttula Ishida), spotted leaf beetle (Epilachna vigintioctopunctata Fab.), shoot and fruit borer (Leucinodes orbonalis Guen.), brinjal leaf beetle (Psylliodes balyi Jacoby) and leaf folder (Eublemma oliracea Walk.) are common pests. The Shoot and Fruit Borer (SFB), Leucinodes orbonalis (Lepidoptera: Pyraustidae) is however a key pest of brinjal throughout the country (Latif et al., 2010; Chakraborti and Sarkar, 2011; Saimandir and Gopal, 2012). In early stage, larva bores into the shoots resulting in drooping, withering and drying of the affected shoots. During fruiting stage, tiny larva bores into the flower buds and fruits, the bored larva are invariably plugged with excreta. The infested fruit become unfit for consumption and market due to loss of quality. The yield loss due to this pest was accounted to the tune of $70-92 \%$ in India (Reddy and Srinivasa, 2004; Jagginavar et al., 2009; Chakraborti and Sarkar, 2011). The SFB has also been reported as a major pest of brinjal in Meghalaya; caused 26.3-62.5\% fruit damage (Gangwar and Sachan, 1981). Many conventional and synthetic pesticides are being used for the management of this notorious pest. Nevertheless, SFB has developed resistance against wide range of conventional pesticides; resulting into the need 
of higher doses of pesticides for their management; which increases the environmental pollution. Keeping these views in mind, the present experiment was conducted to evaluate the some bio-pesticides and newly introduced insecticides with novel mode of action for effective management of brinjal shoot and fruit borer.

\section{Materials and Methods}

The field experiments were conducted at ICAR Research Complex for North-Eastern Hill Region, Umiam, Meghalaya, India during 2011-2012 to evaluate some bio-pesticides and novel insecticides against SFB of brinjal. The brinjal (variety: Ri-Bhoi Local) seedlings were transplanted into the plot size of $5 \times 4 \mathrm{~m}^{2}$ area with a spacing of $75 \times 60 \mathrm{~cm}^{2}$. The experiments were laid out in Randomized Block Design (RBD) with three replications. The treatments viz., azadirachtin 1 EC $(2 \mathrm{ml}$ $\left.\mathrm{l}^{-1}\right)$, karanjin $2 \mathrm{EC}\left(2 \mathrm{ml} \mathrm{l}^{-1}\right)$, anonin $1 \mathrm{EC}\left(2 \mathrm{ml} \mathrm{l}^{1^{-1}}\right)$, Bacillus thuringiensis (Bt.) $\left(2 \mathrm{~g} \mathrm{l}^{-1}\right)$, emamectin benzoate $5 \mathrm{SG}(0.4$ $\left.\mathrm{ml} \mathrm{l}^{-1}\right)$, flubendiamide $480 \mathrm{SC}\left(0.3 \mathrm{ml} \mathrm{l}^{-1}\right)$, chlorantraniliprole 18.5 SC $\left(0.4 \mathrm{ml} \mathrm{l}^{-1}\right)$, chlorpyriphos $20 \mathrm{EC}\left(2 \mathrm{ml} \mathrm{l}^{-1}\right)$ were applied thrice at fifteen days interval starting from initiation of SFB infestation. Spraying was done by pneumatic knapsack sprayer using spray fluid @350 lit ha ${ }^{-1}$. Shoot infestation was recorded from five randomly selected tagged plants from each plot on 1 day before and on 7,14 days after each spray. Numbers of infested and healthy fruits were recorded after each picking from each replicated plot. Weight of healthy and damaged fruit was recorded from each plot separately. Mean shoot and fruit infestation and yield of brinjal were calculated for statistical analysis. Then data were subjected to suitable transformation and the critical difference $\mathrm{CD}(p=0.05)$ level of significance was worked out by one way ANOVA.

\section{Results and Discussion}

Effects of bio-pesticides and other insecticides on shoot and fruit infestation and on yield are presented in Table 1,2 and 3 , respectively.

\subsection{Effects of insecticidal treatments on shoot infestation of} brinjal during 2011-2012

During first year (i.e. 2011), there was a significant difference in shoot infestation in the insecticidal treatments (Table 1). Lowest mean shoot infestation was recorded in chlorantraniliprole treated plots (3.12\%) which was statistically at par with flubendiamide treatment $(3.66 \%)$. Next best treatment in reducing shoot infestation was emamectin benzoate (4.49\%) which was at par with Bacillus thuringiensis (5.98\%) and chlorpyriphos $(6.19 \%)$ treatments. Botanical pesticides such as azadirachtin $(9.66 \%)$, karanjin $(11.87 \%)$ and annonin $(10.13 \%)$ were not as effective as other treatments but all treatments were statistically superior over untreated control plots (17.06\%). During 2012, trend of shoot infestation was almost similar with the first year infestation (Table 1). Minimum shoot infestation was found in chlorantraniliprole treated plots $(1.81 \%)$ which was at par with flubendiamide $(2.49 \%)$ and emamectin benzoate (3.13\%) treatments. Among bio-pesticide, B. thuringiensis showed its superiority over others and it was at par with conventional insecticide, chlorpyriphos (3.95\%). Next best treatment in reducing shoot infestation was emamectin benzoate (4.49\%). Azadirachtin, karanjin and annonin showed similar effectiveness in reducing shoot infestation. Mean of two year experimental results showed that chlorantraniliprole (2.46\%) was the best treatment in reducing the shoot infestation (81.88\% reduction) which was statistically at par with flubendiamide (3.08\%) and emamectin benzoate $(3.76 \%)$ with $77.37 \%$ and $71.95 \%$ reduction over untreated control, respectively. Chlorpyriphos (4.97\%) and B. thuringiensis $(5.32 \%)$ were at par each other with $62.68 \%$ and $62.07 \%$ reduction over untreated control, respectively. Annonin, karanjin and azadirachtin were less effective in reducing shoot borer infestation but all these were statistically superior over untreated control.

\subsection{Effects of insecticidal treatments on fruit infestation of brinjal during 2011-2012}

Effects of insecticidal treatments on fruit infestation are depicted in (Table 2). Significant difference was observed among the treatments in reducing fruit infestation during both the years (Table 2). During 2011, chlorantraniliprole recorded lowest mean fruit infestation $(7.20 \%)$ which was at par with flubendiamide (7.96\%) and emamectin benzoate $(10.05 \%)$. Next best treatments were chlorpyriphos and B. thuringiensis with 15.72 and $15.64 \%$ fruit infestation, respectively. Botanical pesticides were not much superior as compared to others treatments in reducing fruit infestation but these were better over untreated control. During 2012, lowest fruit damage was recorded in flubendiamide treated plots $(3.90 \%)$ which was statistically at par with chlorantraniliprole treatment $(4.32 \%)$. Emamectin benzoate was the next best treatment (8.46\%) followed by chlorpyriphos $(10.80 \%)$ and $B$. thuringiensis $(11.25 \%)$. Among botanicals, annonin recorded less fruit infestation (16.12\%) followed by neem (18.34\%) and karanjin (20.51\%). As per overall mean fruit infestation, chlorantraniliprole $(5.76 \%)$ and flubendiamide (5.93\%) were found to be very effective insecticides in reducing fruit infestation with $79.45 \%$ and $78.84 \%$ reduction over untreated plots, respectively. Though, these insecticides were at par with emamectin benzoate $(9.26 \%)$ with $66.98 \%$ reduction of fruit damage over untreated control plots. Chlorpyriphos $(13.26 \%)$ and B. thuringiensis (13.45\%) showed almost similar effectiveness against fruit borer damage with $52.69 \%$ and $52.02 \%$ reduction over untreated control plots. Botanicals pesticides were found to be superior over untreated control plots but substandard as compared to other treatments. 


\begin{tabular}{|c|c|c|c|c|c|c|c|c|c|c|c|}
\hline \multirow[t]{3}{*}{ Treatment } & \multirow{3}{*}{$\begin{array}{c}\text { Dose } \\
\text { ( } \mathrm{ml} \mathrm{or}^{-1}\end{array}$} & \multicolumn{8}{|c|}{ Mean shoot infestation (\%) after each spray by Leucinodes orbonalis Guen } & \multirow{3}{*}{$\begin{array}{c}\text { Overall } \\
\text { mean shoot } \\
\text { infestation } \\
(\%)\end{array}$} & \multirow{3}{*}{$\begin{array}{c}\text { Protec- } \\
\text { tion over } \\
\text { control } \\
(\%)\end{array}$} \\
\hline & & \multicolumn{4}{|c|}{2011} & \multicolumn{4}{|c|}{2012} & & \\
\hline & & $\begin{array}{c}\text { I } \\
\text { Spray }\end{array}$ & $\begin{array}{c}\text { II } \\
\text { Spray }\end{array}$ & $\begin{array}{c}\text { III } \\
\text { Spray }\end{array}$ & Mean & $\begin{array}{c}\text { I } \\
\text { Spray }\end{array}$ & $\begin{array}{c}\text { II } \\
\text { Spray }\end{array}$ & $\begin{array}{c}\text { III } \\
\text { Spray }\end{array}$ & Mean & & \\
\hline $\begin{array}{l}\text { Azadirachtin } \\
1 \mathrm{EC}\end{array}$ & $\begin{array}{c}2 \mathrm{ml} \\
\mathrm{l}^{-1}\end{array}$ & $\begin{array}{c}10.33 \\
(18.75)\end{array}$ & $\begin{array}{c}10.20 \\
(18.59)\end{array}$ & $\begin{array}{c}8.45 \\
(16.76)\end{array}$ & $\begin{array}{c}9.66 \\
(18.09)\end{array}$ & $\begin{array}{c}7.13 \\
(15.42)\end{array}$ & $\begin{array}{c}5.28 \\
(13.25)\end{array}$ & $\begin{array}{c}4.88 \\
(12.63)\end{array}$ & $\begin{array}{c}5.76 \\
(13.82)\end{array}$ & $\begin{array}{c}7.71 \\
(16.09)\end{array}$ & 43.28 \\
\hline Karanjin 2 EC & $\begin{array}{c}2 \mathrm{ml} \\
\mathrm{l}^{-1}\end{array}$ & $\begin{array}{c}14.24 \\
(22.14)\end{array}$ & $\begin{array}{c}12.05 \\
(20.27)\end{array}$ & $\begin{array}{c}9.32 \\
(17.77)\end{array}$ & $\begin{array}{c}11.87 \\
(20.14)\end{array}$ & $\begin{array}{c}6.71 \\
(14.98)\end{array}$ & $\begin{array}{c}6.25 \\
(14.43)\end{array}$ & $\begin{array}{c}5.37 \\
(13.39)\end{array}$ & $\begin{array}{c}6.11 \\
(14.29)\end{array}$ & $\begin{array}{c}8.99 \\
(17.43)\end{array}$ & $33.87 x$ \\
\hline Annonin $1 \mathrm{EC}$ & $\begin{array}{c}2 \mathrm{ml} \\
\mathrm{l}^{-1}\end{array}$ & $\begin{array}{c}12.89 \\
(21.00)\end{array}$ & $\begin{array}{c}10.82 \\
(19.19)\end{array}$ & $\begin{array}{c}6.68 \\
(14.97)\end{array}$ & $\begin{array}{c}10.13 \\
(18.55)\end{array}$ & $\begin{array}{c}8.23 \\
(16.55)\end{array}$ & $\begin{array}{c}5.98 \\
(13.97)\end{array}$ & $\begin{array}{c}4.56 \\
(12.31)\end{array}$ & $\begin{array}{c}6.26 \\
(14.44)\end{array}$ & $\begin{array}{c}8.19 \\
(16.61)\end{array}$ & 39.73 \\
\hline Bt. $8 \mathrm{~L}$ & $2 \mathrm{~g} \mathrm{l}^{-1}$ & $\begin{array}{c}7.55 \\
(15.81)\end{array}$ & $\begin{array}{c}6.05 \\
(14.18)\end{array}$ & $\begin{array}{c}4.35 \\
(12.01)\end{array}$ & $\begin{array}{c}5.98 \\
(14.13)\end{array}$ & $\begin{array}{c}5.43 \\
(13.46)\end{array}$ & $\begin{array}{c}3.65 \\
(10.82)\end{array}$ & $\begin{array}{c}3.91 \\
(11.40)\end{array}$ & $\begin{array}{c}4.33 \\
(12.01)\end{array}$ & $\begin{array}{c}5.32 \\
(13.12)\end{array}$ & 62.07 \\
\hline $\begin{array}{l}\text { Emamectin } \\
\text { Benzoate } 5 \mathrm{SG}\end{array}$ & $\begin{array}{l}0.4 \mathrm{~g} \\
\mathrm{l}^{-1}\end{array}$ & $\begin{array}{c}5.67 \\
(13.56)\end{array}$ & $\begin{array}{c}5.25 \\
(13.14)\end{array}$ & $\begin{array}{c}2.56 \\
(9.15)\end{array}$ & $\begin{array}{c}4.49 \\
(12.22)\end{array}$ & $\begin{array}{c}3.97 \\
(11.49)\end{array}$ & $\begin{array}{c}3.01 \\
(9.84)\end{array}$ & $\begin{array}{c}2.42 \\
(8.92)\end{array}$ & $\begin{array}{c}3.13 \\
(10.16)\end{array}$ & $\begin{array}{c}3.76 \\
(11.26)\end{array}$ & 71.95 \\
\hline $\begin{array}{l}\text { Flubendiamide } \\
480 \mathrm{SC}\end{array}$ & $\begin{array}{c}0.3 \\
\mathrm{ml} \mathrm{l}^{-1}\end{array}$ & $\begin{array}{c}5.08 \\
(12.99)\end{array}$ & $\begin{array}{c}3.96 \\
(11.33)\end{array}$ & $\begin{array}{l}1.95 \\
(7.97)\end{array}$ & $\begin{array}{c}3.66 \\
(10.99)\end{array}$ & $\begin{array}{c}3.64 \\
(10.92)\end{array}$ & $\begin{array}{c}1.96 \\
(8.02)\end{array}$ & $\begin{array}{c}1.87 \\
(7.85)\end{array}$ & $\begin{array}{c}2.49 \\
(9.06)\end{array}$ & $\begin{array}{c}3.08 \\
(10.10)\end{array}$ & 77.37 \\
\hline $\begin{array}{l}\text { Chlorantrani- } \\
\text { liprole } 18.5 \mathrm{SC}\end{array}$ & $\begin{array}{c}0.4 \\
\mathrm{ml} \mathrm{l}^{-1}\end{array}$ & $\begin{array}{c}4.95 \\
(12.79)\end{array}$ & $\begin{array}{c}3.25 \\
(10.35)\end{array}$ & $\begin{array}{l}1.15 \\
(6.15)\end{array}$ & $\begin{array}{c}3.12 \\
(10.15)\end{array}$ & $\begin{array}{c}2.67 \\
(9.23)\end{array}$ & $\begin{array}{c}1.60 \\
(7.22)\end{array}$ & $\begin{array}{l}1.16 \\
(6.14)\end{array}$ & $\begin{array}{c}1.81 \\
(7.64)\end{array}$ & $\begin{array}{c}2.46 \\
(9.01)\end{array}$ & 81.88 \\
\hline $\begin{array}{l}\text { Chlorpyriphos } \\
20 \text { EC }\end{array}$ & $\begin{array}{c}2 \mathrm{ml} \\
\mathrm{l}^{-1}\end{array}$ & $\begin{array}{c}7.83 \\
(16.22)\end{array}$ & $\begin{array}{c}5.85 \\
(13.95)\end{array}$ & $\begin{array}{c}4.90 \\
(12.77)\end{array}$ & $\begin{array}{c}6.19 \\
(14.39)\end{array}$ & $\begin{array}{c}4.85 \\
(12.52)\end{array}$ & $\begin{array}{c}4.15 \\
(11.69)\end{array}$ & $\begin{array}{c}2.86 \\
(9.68)\end{array}$ & $\begin{array}{c}3.95 \\
(11.38)\end{array}$ & $\begin{array}{c}4.97 \\
(12.98)\end{array}$ & 62.68 \\
\hline Control & - & $\begin{array}{c}15.25 \\
(22.96)\end{array}$ & $\begin{array}{c}19.16 \\
(25.88)\end{array}$ & $\begin{array}{c}16.76 \\
(24.14)\end{array}$ & $\begin{array}{c}17.06 \\
(24.37)\end{array}$ & $\begin{array}{c}8.05 \\
(16.40)\end{array}$ & $\begin{array}{c}10.43 \\
(18.83)\end{array}$ & $\begin{array}{c}11.92 \\
(20.14)\end{array}$ & $\begin{array}{c}10.13 \\
(18.53)\end{array}$ & $\begin{array}{c}13.60 \\
(21.61)\end{array}$ & - \\
\hline $\operatorname{SEm} \pm$ & - & 1.03 & 1.11 & 0.78 & 0.60 & 1.05 & 1.01 & 0.70 & 0.66 & 0.50 & - \\
\hline $\mathrm{CD}(p=0.05)$ & - & 3.09 & 3.33 & 2.34 & 1.78 & 3.14 & 3.03 & 2.10 & 1.98 & 1.49 & - \\
\hline
\end{tabular}

Figures in the parenthesis are angular transformed values

3.3. Effects of insecticidal treatments on yield of brinjal during 2011-2012

Yield of brinjal for the year 2011 and 2012 is presented in (Table 3). During 2011, highest marketable yield (147.56 $\mathrm{q} \mathrm{ha}^{-1}$ ) was found in chlorantraniliprole treated plots. Next best treatment was flubendiamide which recorded $140.45 \mathrm{q}$ ha $^{-1}$ marketable fruit yield followed by emamectin benzoate (126.20 q ha ${ }^{-1}$ ) and chlorpyriphos (117.35 q ha-1). Similar trend of marketable fruit yield of brinjal was also observed during 2012. Pooled of two years experiments indicated that highest marketable yield was recorded in chlorantraniliprole treated plots $\left(155.01 \mathrm{q} \mathrm{ha}^{-1}\right)$ followed by flubendiamide (149.50 q ha-1), emamectin benzoate (134.24 q ha-1) and chlorpyriphos (125.43 q ha-1). Among bio-pesticides, highest mean marketable yield was recorded in Bacillus thuringiensis treated plots (114.45 $\left.\mathrm{q} \mathrm{ha}^{-1}\right)$ followed by neem $\left(101.11 \mathrm{q} \mathrm{ha}^{-1}\right)$.

In the present investigation, chlorantraniliprole and flubendiamide provided excellent results in reducing the shoot and fruit borer infestation during both the years. Devi et al. (2014) reported that out of seven insecticidal treatments, rynaxypyr (chlorantraniliprole) was effective and significantly superior over other treatments in controlling shoot and fruit borer incidence. Efficacy of rynaxypyr (chlorantraniliprole) against brinjal fruit and shoot borer are in close conformity with earlier work (Nayak et al., 2011; Misra, 2011). Effectiveness of rynaxypyr against lepidopteran pests was already reported earlier by many authors (Chowdary et al., 2010; Boselli and Ceredi, 2010). The efficacy of flubendiamide is similar with the results of many researchers (Latif et al., 2010; Jagginavar et al., 2009) who also reported that flubendiamide was most effective against Leucinodes orbonalis. Efficacy of these insecticides are in conformity with Chakraborti and Sarkar (2011) who reported that after two need-based applications of new generation pesticide molecules like flubendiamide or rynaxypyr or emamectin benzoate were produced healthy yields. Chlorantraniliprole and flubendamide proved their superiority over other insecticides in reducing infestation of $L$. orbonalis and resulted in higher yields (Shirale et al., 2012). Results of emamectin benzoate are in agreement with Anil and Sharma (2010) who reported that emamectin benzoate was highly effective treatment against shoot and fruit borer of brinjal. Chlorpyriphos although reduced shoot and fruit infestation of eggplant and protected higher yield as compared to control, its effectiveness was not satisfactory as in newer insecticides. Similar results of chlorpyriphos against 


\begin{tabular}{|c|c|c|c|c|c|c|c|c|c|c|c|}
\hline \multirow[t]{3}{*}{ Treatment } & \multirow{3}{*}{$\begin{array}{c}\text { Dose } \\
(\mathrm{ml} \text { or } \\
\mathrm{g} \mathrm{l}^{-1} \text { ) }\end{array}$} & \multicolumn{8}{|c|}{$\begin{array}{l}\text { Mean fruit infestation (\%) at fortnightly intervals by } \\
\text { Leucinodes orbonalis Guen }\end{array}$} & \multirow{3}{*}{$\begin{array}{c}\text { Overall } \\
\text { mean fruit } \\
\text { infestation } \\
(\%)\end{array}$} & \multirow{3}{*}{$\begin{array}{c}\text { Protec- } \\
\text { tion over } \\
\text { control } \\
(\%)\end{array}$} \\
\hline & & \multicolumn{4}{|c|}{2011} & \multicolumn{4}{|c|}{2012} & & \\
\hline & & First & Second & Third & Mean & First & Second & Third & Mean & & \\
\hline $\begin{array}{l}\text { Azadirachtin } \\
1 \mathrm{EC}\end{array}$ & $\begin{array}{c}2 \mathrm{ml} \\
1^{-1}\end{array}$ & $\begin{array}{c}19.91 \\
(26.48)\end{array}$ & $\begin{array}{l}21.78 \\
(27.80)\end{array}$ & $\begin{array}{c}23.56 \\
(29.03)\end{array}$ & $\begin{array}{c}21.75 \\
(27.76)\end{array}$ & $\begin{array}{c}16.72 \\
(24.11)\end{array}$ & $\begin{array}{c}17.84 \\
(24.94)\end{array}$ & $\begin{array}{c}20.46 \\
(26.85)\end{array}$ & $\begin{array}{c}18.34 \\
(25.33)\end{array}$ & $\begin{array}{l}20.05 \\
(26.57)\end{array}$ & 28.47 \\
\hline $\begin{array}{l}\text { Karanjin } \\
2 \mathrm{EC}\end{array}$ & $\begin{array}{c}2 \mathrm{ml} \\
\mathrm{l}^{-1}\end{array}$ & $\begin{array}{c}20.32 \\
(26.77)\end{array}$ & $\begin{array}{c}22.95 \\
(28.59)\end{array}$ & $\begin{array}{c}25.91 \\
(30.58)\end{array}$ & $\begin{array}{c}23.06 \\
(28.65)\end{array}$ & $\begin{array}{c}17.56 \\
(24.71)\end{array}$ & $\begin{array}{c}20.42 \\
(26.85)\end{array}$ & $\begin{array}{c}23.55 \\
(29.01)\end{array}$ & $\begin{array}{c}20.51 \\
(26.92)\end{array}$ & $\begin{array}{c}21.79 \\
(27.80)\end{array}$ & 22.27 \\
\hline $\begin{array}{l}\text { Annonin } \\
1 \mathrm{EC}\end{array}$ & $\begin{array}{c}2 \mathrm{ml} \\
\mathrm{l}^{-1}\end{array}$ & $\begin{array}{c}17.25 \\
(24.50)\end{array}$ & $\begin{array}{c}20.94 \\
(27.19)\end{array}$ & $\begin{array}{c}23.46 \\
(28.92)\end{array}$ & $\begin{array}{c}20.55 \\
(26.92)\end{array}$ & $\begin{array}{c}14.83 \\
(22.60)\end{array}$ & $\begin{array}{c}15.67 \\
(23.28)\end{array}$ & $\begin{array}{c}17.86 \\
(24.98)\end{array}$ & $\begin{array}{c}16.12 \\
(23.67)\end{array}$ & $\begin{array}{c}18.34 \\
(25.29)\end{array}$ & 34.58 \\
\hline Bt. $8 \mathrm{~L}$ & $2 \mathrm{~g} \mathrm{l}^{-1}$ & $\begin{array}{c}13.17 \\
(21.23)\end{array}$ & $\begin{array}{l}14.78 \\
(22.58)\end{array}$ & $\begin{array}{c}18.97 \\
(25.77)\end{array}$ & $\begin{array}{c}15.64 \\
(23.26)\end{array}$ & $\begin{array}{c}9.52 \\
(17.93)\end{array}$ & $\begin{array}{c}11.78 \\
(20.03)\end{array}$ & $\begin{array}{c}12.45 \\
(20.66)\end{array}$ & $\begin{array}{c}11.25 \\
(19.60)\end{array}$ & $\begin{array}{l}13.45 \\
(21.49)\end{array}$ & 52.02 \\
\hline $\begin{array}{l}\text { Emamectin } \\
\text { Benzoate } 5 \mathrm{SG}\end{array}$ & $\begin{array}{l}0.4 \mathrm{~g} \\
\mathrm{l}^{-1}\end{array}$ & $\begin{array}{c}10.74 \\
(19.12)\end{array}$ & $\begin{array}{c}10.04 \\
(18.43)\end{array}$ & $\begin{array}{c}9.37 \\
(17.81)\end{array}$ & $\begin{array}{c}10.05 \\
(18.42)\end{array}$ & $\begin{array}{c}9.97 \\
(18.39)\end{array}$ & $\begin{array}{c}8.46 \\
(16.90)\end{array}$ & $\begin{array}{c}6.95 \\
(15.24)\end{array}$ & $\begin{array}{c}8.46 \\
(16.89)\end{array}$ & $\begin{array}{c}10.65 \\
(19.02)\end{array}$ & 66.98 \\
\hline $\begin{array}{l}\text { Flubendiamide } \\
480 \mathrm{SC}\end{array}$ & $\begin{array}{c}0.3 \mathrm{ml} \\
\mathrm{l}^{-1}\end{array}$ & $\begin{array}{c}10.73 \\
(19.09)\end{array}$ & $\begin{array}{c}7.23 \\
(15.54)\end{array}$ & $\begin{array}{c}5.92 \\
(14.01)\end{array}$ & $\begin{array}{c}7.96 \\
(16.35)\end{array}$ & $\begin{array}{c}4.85 \\
(12.72)\end{array}$ & $\begin{array}{c}3.17 \\
(10.26)\end{array}$ & $\begin{array}{c}3.67 \\
(11.00)\end{array}$ & $\begin{array}{c}3.90 \\
(11.39)\end{array}$ & $\begin{array}{c}5.93 \\
(14.09)\end{array}$ & 78.84 \\
\hline $\begin{array}{l}\text { Chlorantrani- } \\
\text { liprole } 18.5 \mathrm{SC}\end{array}$ & $\begin{array}{c}0.4 \mathrm{ml} \\
\mathrm{l}^{-1}\end{array}$ & $\begin{array}{c}9.87 \\
(18.20)\end{array}$ & $\begin{array}{c}7.65 \\
(16.04)\end{array}$ & $\begin{array}{c}4.08 \\
(11.60)\end{array}$ & $\begin{array}{c}7.20 \\
(15.51)\end{array}$ & $\begin{array}{c}5.90 \\
(13.98)\end{array}$ & $\begin{array}{c}3.64 \\
(10.98)\end{array}$ & $\begin{array}{c}3.42 \\
(10.54)\end{array}$ & $\begin{array}{c}4.32 \\
(11.99)\end{array}$ & $\begin{array}{c}6.48 \\
(14.72)\end{array}$ & 79.45 \\
\hline $\begin{array}{l}\text { Chlorpyriphos } \\
20 \text { EC }\end{array}$ & $\begin{array}{c}2 \mathrm{ml} \\
\mathrm{l}^{-1}\end{array}$ & $\begin{array}{l}15.16 \\
(22.85)\end{array}$ & $\begin{array}{c}14.52 \\
(22.35)\end{array}$ & $\begin{array}{c}17.48 \\
(24.69)\end{array}$ & $\begin{array}{c}15.72 \\
(23.35)\end{array}$ & $\begin{array}{c}9.92 \\
(18.32)\end{array}$ & $\begin{array}{c}9.72 \\
(18.13)\end{array}$ & $\begin{array}{c}12.76 \\
(20.92)\end{array}$ & $\begin{array}{c}10.80 \\
(19.16)\end{array}$ & $\begin{array}{l}13.26 \\
(21.31)\end{array}$ & 52.69 \\
\hline Control & - & $\begin{array}{c}27.17 \\
(31.41)\end{array}$ & $\begin{array}{c}31.76 \\
(34.29)\end{array}$ & $\begin{array}{c}42.62 \\
(40.75)\end{array}$ & $\begin{array}{c}33.85 \\
(35.56)\end{array}$ & $\begin{array}{c}18.15 \\
(25.22)\end{array}$ & $\begin{array}{c}21.64 \\
(27.72)\end{array}$ & $\begin{array}{c}26.82 \\
(31.19)\end{array}$ & $\begin{array}{c}22.20 \\
(28.11)\end{array}$ & $\begin{array}{l}28.03 \\
(31.97)\end{array}$ & - \\
\hline $\mathrm{SEm} \pm$ & - & 1.02 & 0.99 & 1.05 & 1.14 & 1.10 & 0.92 & 0.70 & 0.54 & 1.20 & - \\
\hline $\mathrm{CD}(p=0.05)$ & - & 3.04 & 2.97 & 3.15 & 3.42 & 3.29 & 2.75 & 2.09 & 1.60 & 3.59 & - \\
\hline
\end{tabular}

Figures in the parenthesis are angular transformed values

Table 3: Effects of insecticidal treatments on yield of brinjal during 2011 to 2012

\begin{tabular}{|c|c|c|c|c|c|c|c|c|c|c|}
\hline \multirow[t]{3}{*}{ Treatment } & \multirow{3}{*}{$\begin{array}{c}\text { Dose } \\
(\mathrm{ml} \text { or } \mathrm{g} \\
\left.\mathrm{1}^{-1}\right)\end{array}$} & \multicolumn{9}{|c|}{ Yield of brinjal $\left(\mathrm{q} \mathrm{ha}^{-1}\right)$} \\
\hline & & \multicolumn{3}{|c|}{ Healthy fruit yield } & \multicolumn{3}{|c|}{ Damaged fruit yield } & \multicolumn{3}{|c|}{ Total fruit yield } \\
\hline & & 2011 & 2012 & Mean & 2011 & 2012 & Mean & 2011 & 2012 & Mean \\
\hline Azadirachtin 1 EC & $2 \mathrm{ml} \mathrm{l}^{-1}$ & 92.55 & 109.67 & 101.11 & 21.43 & 17.18 & 19.31 & 113.98 & 126.85 & 120.42 \\
\hline Karanjin 2 EC & $2 \mathrm{ml} \mathrm{l}^{-1}$ & 85.46 & 98.59 & 92.02 & 23.18 & 19.15 & 21.17 & 108.64 & 117.74 & 113.19 \\
\hline Annonin $1 \mathrm{EC}$ & $2 \mathrm{ml} \mathrm{l}^{-1}$ & 89.92 & 107.33 & 98.62 & 19.56 & 16.39 & 17.98 & 109.48 & 123.72 & 116.60 \\
\hline Bt. $8 \mathrm{~L}$ & $2 \mathrm{~g} \mathrm{l}^{-1}$ & 109.15 & 119.76 & 114.45 & 18.25 & 13.48 & 15.87 & 127.4 & 133.24 & 130.32 \\
\hline Emamectin Benzoate 5 SG & $0.4 \mathrm{~g} \mathrm{l}^{-1}$ & 126.20 & 142.28 & 134.24 & 15.86 & 10.75 & 13.31 & 142.06 & 153.03 & 147.55 \\
\hline Flubendiamide $480 \mathrm{SC}$ & $0.3 \mathrm{ml} \mathrm{l}^{-1}$ & 140.45 & 158.55 & 149.50 & 14.59 & 8.84 & 11.72 & 156.04 & 166.39 & 161.22 \\
\hline Chlorantraniliprole $18.5 \mathrm{SC}$ & $0.4 \mathrm{ml} \mathrm{l}^{-1}$ & 147.56 & 162.47 & 155.01 & 11.26 & 7.92 & 9.59 & 158.82 & 170.39 & 164.61 \\
\hline Chlorpyriphos $20 \mathrm{EC}$ & $2 \mathrm{ml} \mathrm{l}^{-1}$ & 117.35 & 133.52 & 125.43 & 17.45 & 12.89 & 15.17 & 134.8 & 146.41 & 140.61 \\
\hline Control & - & 67.78 & 81.79 & 74.78 & 30.38 & 23.65 & 27.02 & 98.16 & 105.44 & 101.80 \\
\hline $\operatorname{SEm} \pm$ & - & 2.47 & 2.61 & 1.81 & 2.35 & 1.41 & 1.20 & 3.38 & 2.94 & 2.32 \\
\hline $\mathrm{CD}(p=0.05)$ & - & 7.39 & 7.83 & 5.42 & 7.03 & 4.21 & 3.60 & 10.13 & 8.82 & 6.97 \\
\hline
\end{tabular}

brinjal shoot and fruit borer was reported by Latif et al. (2010). The performance of bio-pesticides except Bt. against this pest was the poorest while that of chlorantraniliprole and flubendiamide was the best. Efficacy of $B t$. against brinjal shoot and fruit borer is in agreement with some findings (Patnaik and Singh, 1997; Murali et al., 2002). The effectiveness of azadirectin is disagreement with the findings of Srinivasan and Sundarababu (1998) who reported that 
neem based insecticides were most effective in reducing the incidence of $L$. orbonalis. Results on karanjin are dissimilar with the findings of earlier work where pongamia oil at $1 \%$ to $2 \%$ recorded low fruit borer damage and pongamia oil $2 \%$ recorded highest marketable fruit yield (Reddy and Srinivasa, 2004). Efficacy of annona, karanjin and azadirachtin against insect pests of okra are also reported (Sarkar et al., 2016).

\section{Conclusion}

Chlorantraniliprole and flubendiamide were found to be effective insecticides in reducing the shoot and fruit infestation as well as increasing yield over untreated control. Among bio-pesticides, Bacillus thuringiensis and azadirachtin were also provided satisfactory control of brinjal shoot and fruit borer.

\section{Acknowledgement}

The authors are highly thankful to the Director, ICAR Research Complex for North Eastern Hill Region, Umiam, Meghalaya for providing necessary facilities to conduct the experiment.

\section{References}

Anil, Sharma, P.C., 2010. Bio-efficacy of insecticides against Leucinodes orbonalis on brinjal. Journal of Environmental Biology 31, 399-402.

Anonymous, 2014. Area, production and productivity of fruits and vegetables of different states of the country published by Ministry of Food Processing Industries, Government of India. (http://www.niftem.ac.in/admin/ NewsDocument/01472014024727_011FruitCropWise Data07-08-2014.pdf, Accessed on 03.08.2015).

Boselli, M., Ceredi, G., 2010. Rynaxypyr, new opportunities to control codling moth. Informatore Agrario 66, 43-47.

Chakraborti, S., Sarkar, P.K., 2011. Management of Leucinodes orbonalis Guenee on eggplants during the rainy season in India. Journal of Plant Protection Research 51, 325-328.

Chowdary, L.R., Bheemanna, M., Kumar, L.R., 2010. Bio-efficacy of rynaxypyr (Coragen) $20 \mathrm{SC}$ against fruit borer Helicoverpa armigera (Hubner) in Okra. International Journal of Plant Protection 3, 379-381.

Devi, P., Sahu, T.K., Bihariahirwar, R., Kostha, V.K., 2014. Field evaluation of insecticides for management of shoot and fruit borer, Leucinodes orbonalis Guenee in brinjal. The Ecoscan (6 $6^{\text {th }} \mathrm{Sp}$ Issue), 463-466.

Gangwar, S.K., Sachan, J.N., 1981. Seasonal incidence and control of insect pest of brinjal with special reference to shoot and fruit borer, Leucinodes orbonalis Guen. in Meghalaya. Journal of Research 2, 187-192.

Jagginavar, S.B., Sunitha, N.D., Biradar, A.P., 2009. Bio- efficacy of flubendiamide 480 SC against brinjal fruit and shoot borer, Leucinodes orbonalis Guen. Karnataka Journal of Agricultural Sciences 22, 712-713.

Latif, M.A., Rahman, M.M., Alam, M.Z., 2010. Efficacy of nine insecticides against shoot and fruit borer, Leucinodes orbonalis Guenee (Lepidoptera:Pyralidae) in eggplant. Journal of Pest Science 83, 391-397.

Misra, H.P., 2011. Bio-efficacy of chlorantraniliprole against shoot and fruit borer of brinjal, Leucinodes orbonalis guenee. Journal of Insect Science 24, 60-64.

Murali Krishna, T., Lal, O.P., Srivastava, Y.N.S., Handa, S.K., 2002. Field efficacy of different insecticides, Bacillus thuringiensis var. kurstaki (Bt.), neem and diflubenzuron for the control of shoot and fruit borer, Leucinodes orbonalis Guen. on eggplant. Journal of Entomological Research 26, 43-49.

Nayak, U.S., Baral, K., Rath, L.K., Mandal, P., 2011. Relative efficacy of certain new generation pesticides against brinjal fruit and shoot borer Leucinodes orbonalis Guene. Journal of Plant Protection and Environment 8, 108-109.

NHB, 2015. Indian Horticulture Database-2014 published by National Horticulture Board, Ministry of Agriculture, Government of India.

Patnaik, H.P., Singh, K.M., 1997. Efficacy of Bacillus thuringiensis Berliner and conventional insecticides against brinjal shoot and fruit borer, under different spraying schedules. Orissa Journal of Horticulture 25, 18-21.

Reddy, E., Srinivasa, S.G., 2004. Management of shoot and fruit borer, Leucinodes orbonalis (Guen.) in brinjal using botanicals/oils. Pestology 28, 50-52.

Regupathy, A., Palanisamy, S., Chandramohan, N., Gunathilagaraj, K., 1997. A guide on crop pests. Sooriya desk top publishers, Coimbatore, 264.

Saimandir, J., Gopal, M., 2012. Evaluation of synthetic and natural insecticides for the management of insect pest control of eggplant (Solanum melongena L.) and pesticide residue dissipation pattern. American Journal of Plant Sciences 3, 214-227.

Sarkar, S., Patra, S., Samanta, A., 2016. Efficacy of different bio-pesticides against sucking pests of okra (Abelmoschus esculentus L. Moench) Journal of Applied and Natural Science 8, 333-339.

Shirale, D., Patil, M., Zehr, U., Parimi, S., 2012. Newer insecticides for the management of brinjal fruit and shoot borer, Leucinodes orbonalis. Indian Journal of Plant Protection 40, 273-275.

Srinivasan, G., Sundarababu, P.C., 1998. Management of brinjal shoot and fruit borer Leucinodes orbonalis Guencee (Lepidoptera: Pyralidae) using neem products and insecticides. In: Proceedings of First National Symposium on Pest Management in Horticultural Crop. IIHR, Bangalore, $15^{\text {th }}-17^{\text {th }}$ October, 1997, 87-93. 